\title{
Delayed Correction of Hypotony Maculopathy in a Patient with Glaucoma and Thyroid-Related Orbitopathy
}

\author{
Divakar Gupta $^{\mathrm{a}}$ Mark Slabaugh $^{\mathrm{b}}$ Courtney E. Francis ${ }^{\mathrm{a}}$ \\ ${ }^{a}$ Department of Ophthalmology, University of Washington, Seattle, Wash., and \\ ${ }^{b}$ Department of Ophthalmology and Visual Science, The Ohio State University, \\ Columbus, Ohio, USA
}

\section{Key Words}

Hypotony maculopathy · Optic disc edema - Trabeculectomy · Thyroid-related orbitopathy

\begin{abstract}
Hypotony maculopathy is a condition that may result in visual deterioration from choroidal folds or optic disc edema. Optic disc edema can result from altered translaminar pressure gradients (lowered intraocular pressure, increased cerebrospinal fluid pressure, or increased orbital pressure). We report a case of bilateral optic disc edema in a patient with glaucoma and thyroid-related orbitopathy. The patient's disc edema was initially presumed to be secondary to her orbitopathy, but persisted after bilateral orbital decompression. Ultimately, disc edema and choroidal folds in the right eye resolved after trabeculectomy revision and an increase in intraocular pressure. This case serves as a reminder of the causes of optic disc edema and demonstrates delayed reversal of hypotony maculopathy.
\end{abstract}

\section{Introduction}

Alterations in pressure gradients across the lamina cribrosa may result in optic disc edema when the intraocular pressure is lowered or the retrolaminar pressure is increased. Patients with hypotony, such as those with low intraocular pressure after having undergone trabeculectomy, may have optic disc edema as well as other fundus findings, including retinal vessel tortuosity and choroidal folds. At the same time, patients with thyroid-related orbitopathy or pseudotumor cerebri may have optic disc edema from optic nerve compres-

\begin{tabular}{ll}
\hline KARGER 125/s & $\begin{array}{l}\text { Courtney E. Francis, MD } \\
325 \text { 9th Avenue } \\
\text { PO Box } 359608 \\
\text { Seattle, WA 98104 (USA) } \\
\text { E-Mail francis3@ @w.edu }\end{array}$
\end{tabular}


Gupta et al.: Delayed Correction of Hypotony Maculopathy in a Patient with Glaucoma and Thyroid-Related Orbitopathy

sion and increased orbital or cerebrospinal fluid (CSF) pressure, respectively. In the following case, we describe a patient with bilateral disc edema who had a remote history of bilateral trabeculectomy and who also suffered from thyroid-related orbitopathy. The patient's pathology and surgery altered the translaminar pressure gradients in both eyes. Her clinical course is illustrative of the importance of understanding the causes of optic disc edema.

\section{Case}

A 75-year-old Caucasian woman presented to the neuro-ophthalmology clinic to establish care for thyroid eye disease. She carried a diagnosis of hyperthyroidism and underwent radioiodine ablation 10 years ago. The patient had a complex ocular history, which included a 30-year history of glaucoma initially treated with topical glaucoma medications and argon laser trabeculoplasty and, later, trabeculectomy in both eyes 7.5 years prior to presentation. She has also had cataract surgery in the right and left eyes, 6 and 5 years prior to presentation, respectively.

Before and after cataract surgery in the left eye, the patient was noted on examination to have bilateral 'papillitis' with congestion and hemorrhages of both optic nerves. This was thought to be secondary to thyroid eye disease. Best corrected visual acuity during this time was 20/40 in both eyes, and intraocular pressure ranged from 6 to $9 \mathrm{~mm} \mathrm{Hg}$ in the right eye and from 7 to $11 \mathrm{~mm} \mathrm{Hg}$ in the left eye with no relative afferent pupillary defect. The patient was followed by an outside neuro-ophthalmologist, prescribed oral prednisone, and eventually underwent bilateral orbital decompressions. Optic nerve congestion was thought to improve after orbital decompression, but did not resolve. Residual congestion was thought to be due to prior optic neuropathy, without a current, active process. After decompression, the patient underwent multiple eye lid procedures to correct retraction and ptosis, and she was prescribed prismatic correction for esotropia. A review of outside records showed that 4 years after orbital decompression, her best corrected visual acuity had declined in the right eye from 20/30 to 20/100. In her left eye, her vision had fluctuated between 20/30 and 20/70. Intraocular pressures over this time period ranged from 4 to $8 \mathrm{~mm} \mathrm{Hg}$ in the right eye and from 6 to $8 \mathrm{~mm} \mathrm{Hg}$ in the left, with no relative afferent pupillary defect. Review of an outside 24-2 Humphrey visual field showed an enlarged blind spot in the right eye and mild superior loss in the left eye, without note of progression after orbital decompression.

During initial neuro-ophthalmologic examination at our clinic, the patient's chief complaint was declining vision in both eyes over the past few years. The patient's best corrected visual acuity was 20/80 in the right eye and 20/40 in the left. Intraocular pressure by Goldmann tonometry was $4 \mathrm{~mm} \mathrm{Hg}$ in the right and $6 \mathrm{~mm} \mathrm{Hg}$ in the left eye. Pupil examination revealed poorly reactive pupils bilaterally, with no relative afferent pupillary defect. Motility revealed an approximately $25 \%$ deficit in abduction bilaterally and a 10 prism diopter esotropia in primary gaze. Hertel exophthalmometry was $21 \mathrm{~mm}$ in the right eye and $20 \mathrm{~mm}$ in the left eye.

External examination revealed lid ptosis of the left upper eye lid. Slit lamp examination was significant for bilateral Seidel-negative superior trabeculectomy blebs, superior peripheral iridectomy in both eyes, and pseudophakia of both eyes.

Fundus examination showed bilateral disc edema, not allowing determination of the cup-to-disc ratio, increased vessel tortuosity, macular thickening, and choroidal folds (fig. 1). Macular folds were more prominent in the right eye. No peripheral choroidal detachments were noted. These findings were confirmed on optical coherence tomography (OCT) of the nerve and macula (fig. 1). 
Gupta et al.: Delayed Correction of Hypotony Maculopathy in a Patient with Glaucoma and Thyroid-Related Orbitopathy

These signs suggested hypotony maculopathy in both eyes secondary to overfiltration. Particularly, there was concern for the right eye, which had experienced a steady decline in vision over the past 4 years. The patient was referred to a glaucoma specialist. It was recommended to the patient that she should undergo trabeculectomy revision of the right eye, with scleral patch graft to increase the intraocular pressure. The patient agreed and underwent the procedure. In the immediate postoperative period, the patient's intraocular pressure was poorly controlled in the right eye, rising to $38 \mathrm{~mm} \mathrm{Hg}$. With the introduction of glaucoma eye drops and oral acetazolamide, the pressure improved to mid-teen values. Eventually, the patient was tapered off oral acetazolamide but remained on three topical glaucoma medications (brinzolamide, brimonidine, and latanoprost). Remarkably, 4 weeks after surgery, the patient's visual acuity improved to $20 / 50$ in the right eye and has remained stable for 21 months of follow-up. The patient's visual fields have remained stable, her right optic nerve head edema resolved, and the right macula appeared flat with no folds (fig. 2). Also, the visual acuity, intraocular pressure, and optic disc edema in the left eye have remained stable. The patient declined any surgical intervention in the left eye.

\section{Discussion}

Hypotony maculopathy is most commonly seen after filtering surgery and clinically presents with macular folds, optic disc edema, and hypotony. The use of antimetabolites during filtering surgery, as well as young age, myopia, and a primary filtering procedure are risk factors for hypotony maculopathy [1-3]. Typically, intraocular pressure is $<9 \mathrm{~mm} \mathrm{Hg}$ in hypotony maculopathy; however, there have been cases of maculopathy occurring in patients with higher intraocular pressures [1, 4].

Our patient developed optic disc edema after bilateral trabeculectomy and cataract surgeries, which persisted after orbital decompressions. Optic disc edema results from an increased pressure gradient across the lamina cribrosa. This pressure gradient is formed between the intraorbital CSF and intraocular pressures. After trabeculectomy, optic disc edema most commonly occurs in the setting of hypotony. There have been reports of patients with low-normal intraocular pressure after trabeculectomy who developed optic disc edema. All of these patients had either coexisting pseudotumor cerebri or high-normal CSF pressure, which was thought to have increased their translaminar pressure gradient [4-6]. Only one of these cases resulted in irreversible vision loss [6].

In our case, both trabeculectomy and orbital decompression would have altered the translaminar pressure gradient, with the former increasing outflow and the latter decreasing episcleral venous pressure. After orbital decompression, intraocular pressure is decreased, and gaze-evoked changes in intraocular pressure are dampened [7].

Retrobulbar pressures are also reduced after decompression [8]. The literature provides no data to understand the effect of orbital decompression on intraorbital CSF pressure. We conclude that the sequential trabeculectomy and orbital decompression surgeries altered the translaminar pressure gradient primarily by lowering the intraocular pressure by complimentary mechanisms, leading to persistent optic disc edema independent of intracranial pressure. Curiously, there was some apparent improvement in the optic disc congestion after decompression. The patient, however, had degrading vision, suggesting worsening of her maculopathy at lower intraocular pressures. It is unclear if the patient truly had compressive optic neuropathy requiring orbital decompression, but we suspect that the patient's bilateral disc edema may have been secondary to hypotony alone. 
Gupta et al.: Delayed Correction of Hypotony Maculopathy in a Patient with Glaucoma and Thyroid-Related Orbitopathy

Treatment options for hypotony maculopathy include bandage contact lens tamponade, injection of irritant into the trabeculectomy bleb (such as autologous blood), transconjunctival suturing of the bleb, or revision of the scleral flap. Early intervention has been recommended to prevent deterioration in vision and bleb failure [1]. Delayed correction of hypotony has also been shown to result in improved visual acuity [9]. In our patient, clinical signs of hypotony maculopathy that had been present for at least 4 years resolved with trabeculectomy revision.

In summary, we report a delayed case of hypotony maculopathy in a patient who had trabeculectomy and subsequent orbital decompression. It reminds us to consider the effects of ocular and orbital surgery on intraocular pressure and translaminar pressure gradients. We would recommend to providers to exercise caution during subsequent orbital decompression in patients with previous glaucoma filtering surgery, or filtering surgery after previous orbital decompression, given the potential additive effects of the surgeries on lowering intraocular pressure. This case adds to the body of literature that demonstrates anatomic and functional success even after delayed correction of hypotony.

\section{Acknowledgments}

Supported in part by an unrestricted departmental grant from Research to Prevent Blindness, Inc., New York, N.Y., USA

\section{Statement of Ethics}

The authors have no ethical conflicts to disclose.

\section{Disclosure Statement}

The authors have no conflicts of interest.

\section{References}

1 Suner IJ, Greenfield DS, Miller MP, Nicolela MT, Palmberg PF: Hypotony maculopathy after filtering surgery with mitomycin C. Incidence and treatment. Ophthalmology 1997;104:207-214; discussion 214-215.

-2 Costa VP, Smith M, Spaeth GL, Gandham S, Markovitz B: Loss of visual acuity after trabeculectomy. Ophthalmology 1993;100:599-612.

-3 Stamper RL, McMenemy MG, Lieberman MF: Hypotonous maculopathy after trabeculectomy with subconjunctival 5-fluorouracil. Am J Ophthalmol 1992;114:544-553.

4 Faingold D, Francis CJ, Buys YM: Hypotony maculopathy and papilledema after trabeculectomy in a patient with pseudotumor cerebri. J Glaucoma 2003;12:374-378.

-5 Kawasaki A, Purvin V: Unilateral optic disc edema following trabeculectomy. J Neuroophthalmol 1998;18:121-123.

6 Greenfield DS, Wanichwecharungruang B, Liebmann JM, Ritch R: Pseudotumor cerebri appearing with unilateral papilledema after trabeculectomy. Arch Ophthalmol 1997;115:423-426.

-7 Takahashi Y, Nakamura Y, Ichinose A, Kakizaki H: Intraocular pressure change with eye positions before and after orbital decompression for thyroid eye disease. Ophthal Plast Reconstr Surg 2014;30:47-50.

-8 Otto AJ, Koornneef L, Mourits MP, Deen-van Leeuwen L: Retrobulbar pressures measured during surgical decompression of the orbit. Br J Ophthalmol 1996;80:1042-1045.

-9 Delgado MF, Daniels S, Pascal S, Dickens CJ: Hypotony maculopathy: improvement of visual acuity after 7 years. Am J Ophthalmol 2001;132:931-933. 


\section{Case Reports in Ophthalmology}
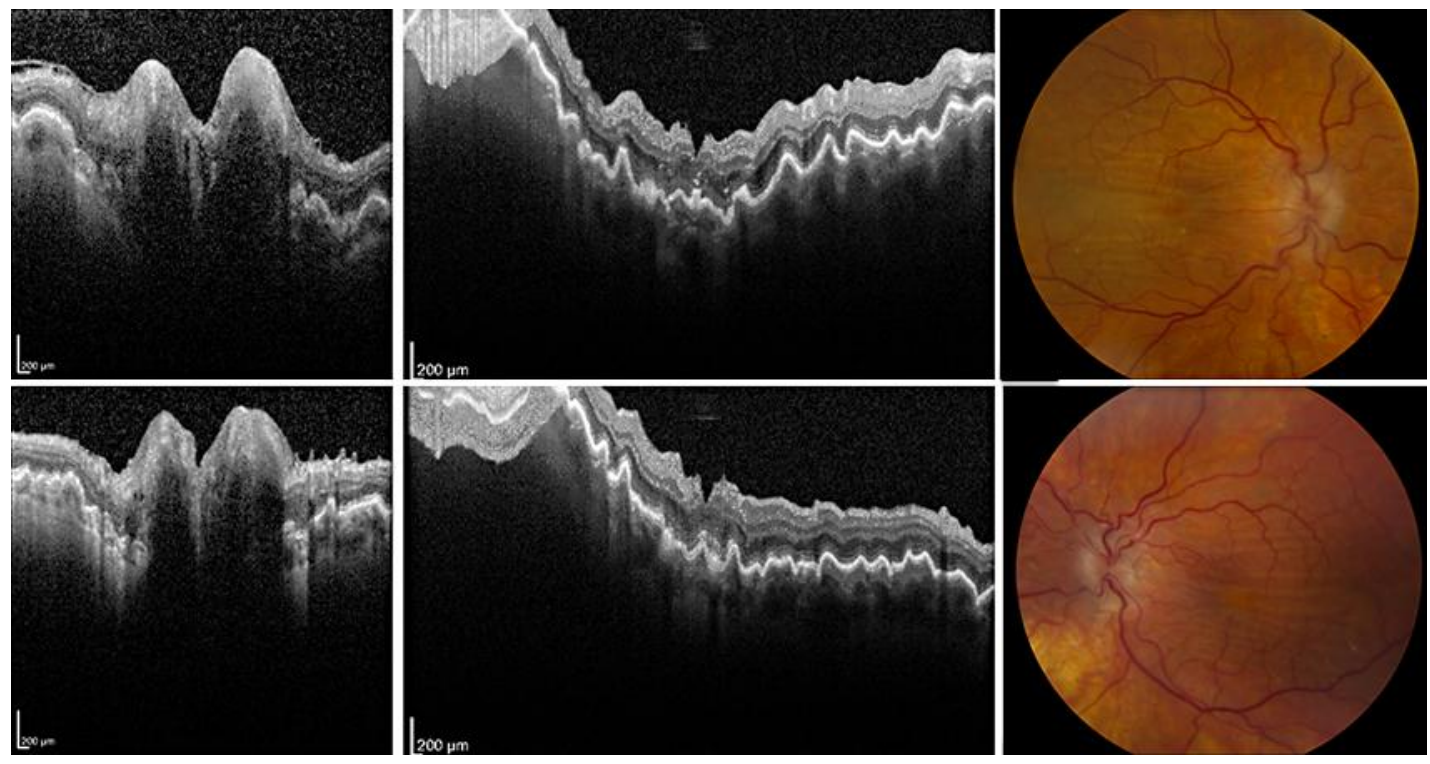

Fig. 1. OCT and fundus photographs showing hypotony maculopathy bilaterally. OCT of the optic nerve head shows edema of the right eye (upper left) and left eye (bottom left). Vertical sections through macula OCT highlight the retinal folds (upper middle: right eye; lower middle: left eye). Color fundus photographs demonstrate similar findings (upper right: right eye; bottom right: left eye).
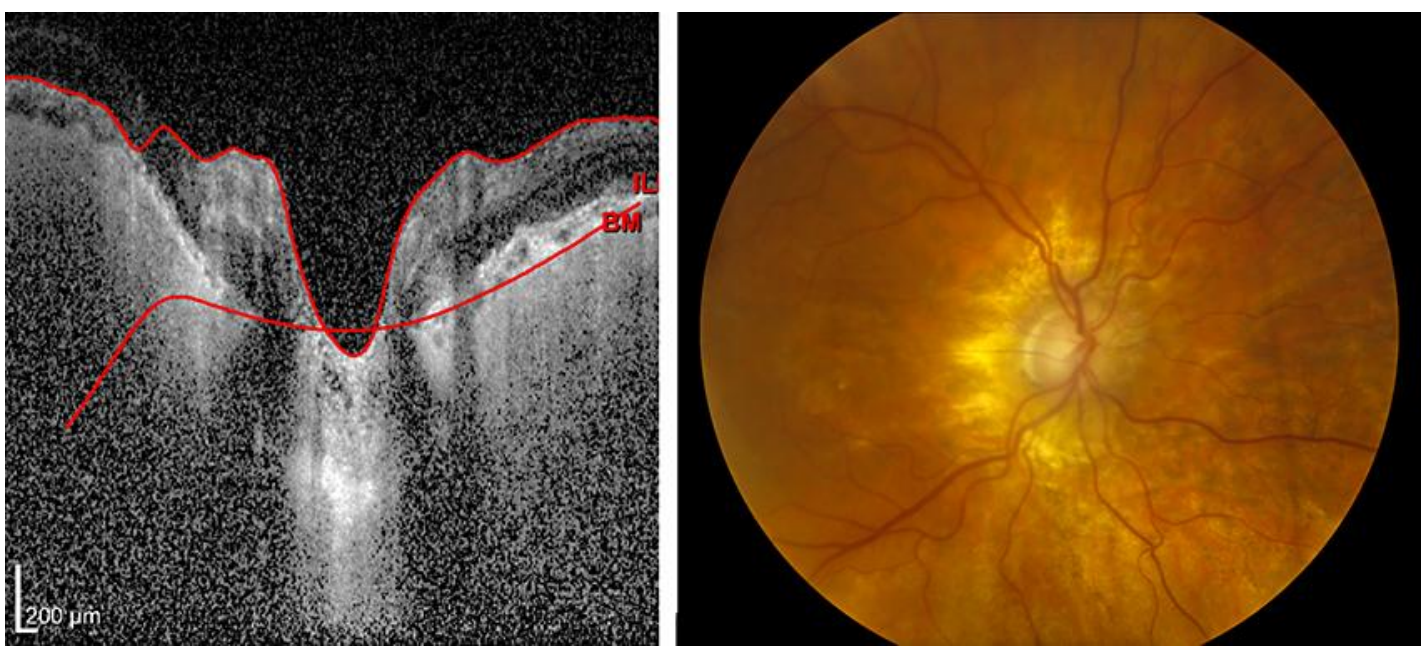

Fig. 2. Resolution of hypotony maculopathy in the right eye. OCT of the optic nerve head shows reformation of the optic cup (left). Fundus photograph showing no evidence of optic disc edema or macular folds (right). 